\title{
Matrix metalloproteinases and chronic rhinosinusitis with nasal polyposis. Unravelling a puzzle through a systematic review*
}

\author{
S. Lygeros' 1 G. Danielides ${ }^{1}$, K. Grafanaki², M. Riga ${ }^{3}$
}

' Department of Otorhinolaryngology-Head and Neck Surgery, University of Patras, Medical School, Patras, Greece

${ }^{2}$ Department of Biochemistry and Department of Dermatology School of Medicine, University of Patras, Greece

${ }^{3}$ Department of Otorhinolaryngology-Head and Neck Surgery, Dammam Medical Complex, Dammam, Kingdom of Saudi Arabia

Rhinology 59: 0, 0 - 0, 2021

https://doi.org/10.4193/Rhin20.578

*Received for publication:

November 12, 2020

Accepted: January 20, 2021

\begin{abstract}
Background: The expression of metalloproteinases (MMPs) in chronic rhinosinusitis with nasal polyposis (CRSwNP) was reviewed in order to investigate their possible use as therapeutical targets and/or biomarkers.
\end{abstract}

Methodology: The differences between CRSwNP and normal controls or CRS without NP, as well as the effects of various treatments on MMPs, tissue inhibitors of MMPs (TIMPs) and MMP/TIMP ratios were considered as primary outcomes. Additional factors reported to affect MMP expression levels were noted as secondary outcomes. Data regarding inflammatory subtypes, patients' clinical characteristics, controls, laboratory method(s) and origin of samples were also pooled. Studies on $\leq 10$ patients or on specimens other than nasal and serum were excluded.

Results: Forty-three studies were included. Tissue sample origin, allergic rhinitis, smoking, infection, medication intake and primary or recurrent disease should be considered as confounding factors for MMP levels. MMP-1 and -7 were consistently found to be significantly higher in CRSwNP patients than controls. CRSWNP endotypes with distinctly different inflammation patterns seem to present similar MMP-related remodelling patterns.

Conclusions: The existing literature has revealed several population and methodology related confounding factors and remains inconclusive regarding the roles of MMPs in CRSwNP pathophysiology and their possible clinical usefulness as biormarkers and therapeutical targets.

Key words: chronic rhinosinusitis, nasal polyposis, matrix metalloproteinase, remodeling, pathophysiology

\section{Introduction}

The role of matrix metalloproteinases (MMPs) and tissue inhibitors of MMPs (TIMPs) in chronic rhinosinusitis (CRS) is currently unclear ${ }^{(1,2)}$. Their physiological and pathophysiological interactions have been recently found to expand well beyond their involvement in the remodelling of extracellular matrix (ECM) and basement membrane, toward the regulation of the release or activation of chemokines, cytokines, growth factors, antibiotic peptides, and other bioactive molecules and toward processes such as innate and adaptive immunity, inflammation, angiogenesis, epithelial-to-mesenchymal transition, bone remodelling and neurite growth ${ }^{(3-7)}$. Their excessive involvement in inflammatory and tissue remodelling processes seems to support the hypothesis of their possible clinical usefulness as biomarkers, predictors of disease severity and/or therapeutical targets in chronic inflammatory diseases with clear tissue remodelling characteristics such as CRS with nasal polyposis (CRSwNP). Similarly, the role of TIMPs has been recently found to exceed a simplistic MMP/TIMP ratio proteolysis model ${ }^{(3)}$. TIMPs can both directly inhibit MMP-induced ECM proteolysis, as well as indirectly control ECM turnover depending on the given local tissue environment (for example injury, disease or infection). Moreover, TIMPs can function in MMP-independent ways ${ }^{(8)}$. The target of the present systematic review is to investigate the 
possible use of MMPs (including a disintegrin and metalloproteinases; ADAMs) and TIMPs as biomarkers and/or therapeutical targets mainly in CRSwNP. The accumulating knowledge on MMPs and TIMPs was reviewed under the prism of recent advances on the pathophysiology and endotype diversity of CRSWNP. Complete descriptions of MMPs and their inhibitors is beyond the scope of this study and can be assessed through excellent reviews ${ }^{(3-7)}$. It is true that the use of MMP inhibitors in the treatment of several other diseases has been so far discouraged by serious adverse effects, as well as by the dual direct or indirect implication of MMPs into both the promotion and inhibition of inflammation, and into both the proteolysis and accumulation of extracellular matrix $(E C M)^{(3-7)}$. However, CRS presents the advantage of being accessible and responsive to local treatments, which could theoretically by-pass the systematic adverse effects of MMP inhibitors. Furthermore, the recent advances toward MMP-and-substrate specific inhibitors might offer target-specific pharmacological options. In both cases, further knowledge of the involvement of MMPs in the pathophysiology of CRS is needed.

\section{Materials and methods}

Eligibility criteria

This review adhered to the recommendations of the PRISMA (Preferred Reporting Items of Systematic Reviews and Meta-analysis) statement ${ }^{(9)}$. Both prospective and retrospective studies addressing the involvement of MMPs in the pathogenesis and preservation of CRSWNP, as well as their potential correlations with CRSwNP clinical, inflammatory and histologic subtypes or characteristics were eligible for inclusion.

Information sources and search

A review of the present literature was conducted via the PubMed database of the US National Library of Medicine (www. pubmed.org). The search terms "metalloproteinase and sinusitis", "MMP and sinusitis", as well as "metalloproteinase and nasal and polyp" limited for the time period 1990- February 2020, written in English, French or German and referring to humans attributed 61,53 and 94 results respectively, in total 208 studies. Earlier studies were not included, because of limitations in the available laboratory methods and in the acquisition of the manuscripts' full texts. No relevant/similar reviews appeared from the search in the Cochrane library.

\section{Study selection}

The studies were initially screened through their abstracts and were excluded if they referred to antrochoanal polyps, silent sinus syndrome, inverted papilloma, cystic fibrosis, primary ciliary dyskinesia, paediatric populations, special subpopulations (such as patients with laryngopharyngeal reflux and cell cultures infected with rhinovirus) or were restricted to chronic

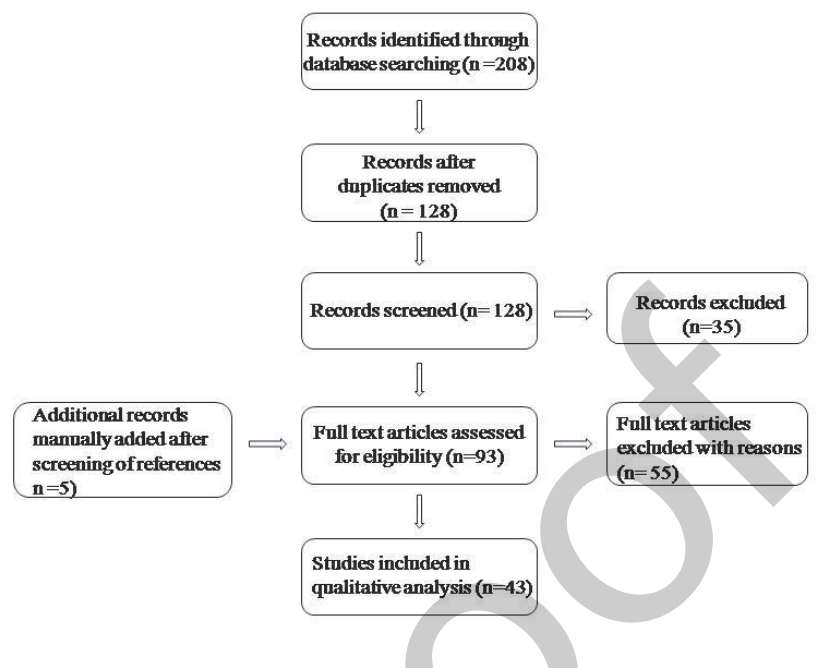

Figure 1. The study selection flowchart.

rhinosinusitis without nasal polyposis (CRSsNP). During the next step, full text manuscripts were screened. The citation lists of included full text studies and relevant narrative reviews were also checked manually. Full text articles or parts of larger studies were excluded in case they referred to MMP detection in specimens other than nasal and serum such as sputum or to effects on MMPs of medications that have been withdrawn. Studies on small populations of $\leq 10$ patients were excluded. This minimum sample size was calculated according to the recommendations for determination of sample size in Health Sciences ${ }^{(10)}$ using the equation $n=\frac{Z^{2} \times \hat{p}(1-\hat{p})}{\varepsilon^{2}}$

for $z$ equivalent to $95 \%$ Confidence Interval, $\varepsilon$ equal to $10 \%$ margin of error (for the calculation and comparison of MMP means in patients and controls) and population proportion ( $p$ ) of $2.5 \%$ for CRSwNP(11). Studies where control groups included or consisted solely of patients diagnosed with other nasal diseases (such as inverted papilloma), studies that compared samples of different origin (for example mucosal tissue with nasal lavage samples) and narrative reviews were also excluded. Results from CRSWNP and CRSsNP subpopulations had to be presented separately. Results on MMP genotyping were beyond the scope of the present review. The study selection flowchart is presented in Figure 1.

\section{Data collection process}

Two investigators independently decided on the eligibility of the studies and extracted data from full text manuscripts. Minor discrepancies were discussed and resolved.

\section{Data items}

We considered as primary outcomes the differences in the protein and/or mRNA expression levels of MMPs, ADAMs, TIMPs and MMP/TIMP ratios between CRSwNP and normal controls or CRSsNP. Factors of heterogeneity between studies were assessed by 
abstracting data on the patients' country of origin, the eosinophilic/non-eosinophilic inflammatory patterns of the samples, the populations used as controls, the diagnostic method(s) used to assess MMP expression levels and the origin of the tissue samples. The effects of various treatment protocols/strategies on the protein and/or mRNA expression levels of MMPs, ADAMs, TIMPs and MMP/TIMP ratios in patients with CRSwNP were also considered as primary outcomes. Any factors reported to affect MMP expression levels were noted as secondary outcomes. Possible correlations between MMPs and clinical characteristics, endoscopic findings, comorbidities or therapeutical results were also pooled. Mean and standard deviation values of MMPs and TIMPs as well as population sizes were used, if extractable, for the quantification of heterogeneity among studies.

\section{Risk of bias in individual studies}

The ongoing research on MPPs has revealed several factors which affect the expression levels of MMPs and the inflammatory processes in the nasal mucosa such as smoking, allergy, asthma, local/systematic use of corticosteroids as well as known MMP inhibitors such as doxycycline, macrolide antibiotics and anti-histamines ${ }^{(12-17)}$. Factors such as asthma and sensitisation to aeroallergens are known to have different prevalence rates among patients with CRSwNP and controls. Consequently, for studies not taking into consideration these parameters (marked with "?" in Table 1) selection bias risks are evident. For studies presenting comparisons between substantially different study groups with regard to parameters currently known to affect MMP levels (marked with " + " in Table 1) confounding bias risks should be considered. Manuscripts were considered as being at low bias risk when they reported the absence or equal administration/diagnosis of the aforementioned parameters between groups (marked with "-" in Table 1).

Selection bias can be estimated based on the prevalence ratio $(P R)^{(18)} . P R=P R S u b / P R T o t$, where PRSub is the prevalence of the factor in CRSwNP patients and PRTot is the prevalence of the factor in controls. A PR equal to 1 indicates no bias. A PR>1 indicates that the bias factor is over-represented in the study group and $\mathrm{PR}<1$ indicates that the factor is under-represented in the study group. A similar method applies for the estimate of individual confounding bias. However, the detailed estimation of the risk of bias in this review is prohibited by the following limitations. First, prevalence rates may vary for different countries thus impeding such a calculation in multicentre studies. Second, for most of the included studies statistical risk calculation requires the combination of multiple confounding factors (Table 1), which possibly interact with each other in yet unknown patterns. The effects of the aforementioned confounding factors toward the overestimation or underestimation of the measured levels of MMPs are presented in the discussion.
Summary measures and synthesis of results

Studies have used different techniques in order to detect protein and/or mRNA levels of MMPs and TIMPs. The literature is highly heterogeneous also regarding study populations, sample origin and selection bias. Review Manager (RevMan) Version 5.4.1 was used to investigate the potential of meta-analyses of the results. Chi-squared $\left(\chi^{2}\right)$ test and $I^{2}$ were used to identify and measure heterogeneity among studies. Within individual studies statistical significance was attributed to two-sided $p<0.05$.

\section{Risk of bias across studies}

The present review is subject to publication bias, since comparisons between patients and controls which attribute nonsignificant results often remain unpublished. Information on the anatomical origin of the examined tissue samples (Table 2), the primary/recurrent CRSwNP history, and the laboratory techniques used were also pooled and considered as possible sources of further risk of bias across studies.

\section{Additional analyses}

Reported correlations between MMPs and specific cytokines such as Thelper-1 (Th1)-related interferon (IFN)- $\gamma$, interleukin (IL)-2 and Tumour Necrosis factor (TNF)- $a$, as well as Th2-related IL-4, IL-5 and IL-13 and Th17-related IL-17 and IL-23 ${ }^{(19-21)}$ were also noted.

\section{Results}

Study selection

The total number of records identified through database searching ( $n=208$ ), was reduced to 128 after removal of duplicates. Abstract screening resulted to the exclusion of 35 studies. Full text manuscripts were therefore acquired for 93 studies, from which the authors excluded with reasons another 55 . Hand searching through the citation lists of included full text studies and narrative reviews resulted to the addition of 5 more studies. Following the aforementioned procedure, results from 43 studies were selected for review and qualitative analysis (Figure 1, Table 1) ${ }^{(13,15,22-62)}$.

\section{Study characteristics}

All studies are prospective and refer to samples taken intraoperatively during FESS (for patient populations) and septoplasty or other nasal or transnasal operations (for controls). Most of the studies have examined tissue samples (Table 2), with considerable heterogeneity regarding their anatomical origin. Significant heterogeneity regarding laboratory techniques was also noted. For most researchers, the method of choice was immunohistochemistry alone or additional to other techniques( ${ }^{(22,26,31,33,34,36,38,39,41-43,49,51,52,57,62)}$. Different methods have been applied for quantifying MMP labelling index. $\operatorname{ELISA}^{(25,27,28,30,32,37,45-47,55,58,60)}$, real time (quantitative) Polymerase 
Table 1. Estimate of bias regarding the presence of confounders in the recruited populations. Manuscripts presenting heterogeneous populations or comparisons between study groups with differences regarding any of the following parameters were marked with + . Studies reporting the absence or equal administration/diagnosis of the following parameters between groups were marked with -. When no information was provided the respective cell was marked with a question mark.

\begin{tabular}{|c|c|c|c|c|c|c|c|c|c|c|}
\hline \multirow{2}{*}{\multicolumn{2}{|c|}{ Study }} & \multicolumn{2}{|c|}{ Steroids } & \multirow{2}{*}{$\begin{array}{l}\text { Mac- } \\
\text { rolides/ } \\
\text { Doxycy- } \\
\text { cline }\end{array}$} & \multirow{2}{*}{$\begin{array}{l}\text { Allergic } \\
\text { rhinitis }\end{array}$} & \multirow{2}{*}{$\begin{array}{l}\text { Smok- } \\
\text { ing }\end{array}$} & \multirow{2}{*}{$\begin{array}{l}\text { Anti- } \\
\text { hista- } \\
\text { mines }\end{array}$} & \multirow[t]{2}{*}{ NERD } & \multirow[t]{2}{*}{ Asthma } & \multirow{2}{*}{$\begin{array}{l}\text { Additional } \\
\text { parameters }\end{array}$} \\
\hline & & Local & $\begin{array}{l}\text { Sys- } \\
\text { temic }\end{array}$ & & & & & & & \\
\hline 1 & $\begin{array}{l}\text { Lechapt-Zalc- } \\
\text { man et al. } 2001^{22}\end{array}$ & - & - & - & $?$ & $?$ & - & $?$ & $?$ & $\begin{array}{l}\text { Controls with vasomotor } \\
\text { rhinitis }\end{array}$ \\
\hline 2 & Liu et al. $2001^{23}$ & - & - & - & - & $?$ & - & - & - & $\begin{array}{l}\text { Fibroblasts cell cultures from } \\
\text { NP and nasal mucosa }\end{array}$ \\
\hline 3 & Lee et al. $2003^{24}$ & $\begin{array}{l}\text { Study of } \\
\text { steroids. }\end{array}$ & elation & between re & modeling & lated $m$ & les ir & sthma & atopic ar & d non-atopic. No topical \\
\hline 4 & $\begin{array}{l}\text { Watelet et al. } \\
2004^{25}\end{array}$ & - & - & - & $+^{*}$ & $?$ & - & $+*$ & $+{ }^{*}$ & $\begin{array}{l}36 \text { pts, } 76 \text { sides in-dependent- } \\
\text { ly studied. Recurrent included. }\end{array}$ \\
\hline 5 & $\begin{array}{l}\text { Chen et al. } \\
2007^{26}\end{array}$ & - & - & - & + & $?$ & ? & & & $\begin{array}{l}\text { Controls with vasomotor } \\
\text { rhinitis }\end{array}$ \\
\hline 6 & $\begin{array}{l}\text { Kostamo et al. } \\
2007^{27}\end{array}$ & $?$ & $?$ & $?$ & + & $?$ & ? & & + & $\begin{array}{l}\text { Mucus from the diseased sinus } \\
\text { of pts vs nasal lavage from } \\
\text { healthy controls }\end{array}$ \\
\hline 7 & Can et al. $2008^{28}$ & - & - & - & $?$ & $?$ & - & $?$ & $?$ & \\
\hline 8 & $\begin{array}{l}\text { Eisenberg et al. } \\
2008^{29}\end{array}$ & - & - & - & - & + & & - & - & No prior surgery \\
\hline 9 & $\begin{array}{l}\text { Kahveci et al. } \\
2008^{30}\end{array}$ & - & - & - & + & $?$ & - & + & + & No prior surgery \\
\hline 10 & Shin et al. $2009^{31}$ & - & - & - & - & $?$ & $?$ & - & - & \\
\hline 11 & $\begin{array}{l}\text { Bugdayci et al. } \\
2010^{32}\end{array}$ & $?$ & $?$ & $?$ & & ? & ? & $?$ & $?$ & \\
\hline 12 & $\begin{array}{l}\text { Erbek et al. } \\
2010^{33}\end{array}$ & - & - & & & $?$ & $?$ & + & + & \\
\hline 13 & Li et al. $2010^{34}$ & - & - & $?$ & - & - & - & - & - & \\
\hline 14 & $\begin{array}{l}\text { Van Zele et al. } \\
2010^{35}\end{array}$ & - & & & & $+*$ & $?$ & $+*$ & $+*$ & $\begin{array}{l}\text { * Baseline MMP-9 among } \\
\text { groups NS in nasal secretions }\end{array}$ \\
\hline 15 & $\begin{array}{l}\text { Eyibilen et al. } \\
2011^{36}\end{array}$ & & & $?$ & + & $?$ & $?$ & + & + & \\
\hline 16 & Yigit et al. $2011^{15}$ & - & - & - & - & - & - & - & - & No controls \\
\hline 17 & $\begin{array}{l}\text { Van Crombrug- } \\
\text { gen et al. } 2012^{37}\end{array}$ & - & 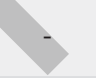 & $?$ & + & $?$ & $?$ & - & + & \\
\hline 18 & $\begin{array}{l}\text { Wang et al. } \\
2012^{38}\end{array}$ & & - & - & - & $?$ & - & - & - & $\begin{array}{l}\text { Recurrent vs non-recurrent } \\
\text { subgroups }\end{array}$ \\
\hline 19 & $\begin{array}{l}\text { de Borja Callejas } \\
\text { et al. } 2013^{39}\end{array}$ & - & - & - & $+{ }^{*}$ & $?$ & $?$ & + & + & Pts of older age $(p=0.005)$ \\
\hline 20 & $\begin{array}{l}\text { Malinsky et al. } \\
2013^{40}\end{array}$ & $?$ & $?$ & $?$ & $?$ & $?$ & ? & $?$ & $?$ & \\
\hline 21 & Shi et al. $2013^{41}$ & - & - & - & - & $?$ & $?$ & $?$ & + & $\begin{array}{l}\text { Atopic vs non-atopic Eosinop- } \\
\text { hilic vs non-eosinophilic NP }\end{array}$ \\
\hline 22 & $\begin{array}{l}\text { Wang et al. } \\
2013^{42}\end{array}$ & - & - & $?$ & $?$ & $?$ & $?$ & $?$ & - & $\begin{array}{l}\text { Study on recurrent CRS. Con- } \\
\text { trols with chronic rhinitis }\end{array}$ \\
\hline 23 & Yeo et al. $2013^{43}$ & - & - & $?$ & - & - & $?$ & - & - & No prior nasal surgery. \\
\hline 24 & $\begin{array}{l}\text { de Borja Callejas } \\
\text { et al. } 2015^{44}\end{array}$ & $\#$ & $\#$ & - & $?$ & $?$ & - & $\begin{array}{c}\text { NS } \\
\text { between } \\
\text { sub-group }\end{array}$ & - & $\begin{array}{l}\text { \#Repeated measures after } \\
\text { treatment }\end{array}$ \\
\hline 25 & $\begin{array}{l}\text { Katainen et al. } \\
2015^{45}\end{array}$ & ? & $?$ & $?$ & - & $?$ & ? & - & - & $\begin{array}{l}\text { Only subgroups with }>10 \mathrm{pts} \\
\text { presented }\end{array}$ \\
\hline 26 & Li et al. $2015^{46}$ & - & - & $?$ & $?$ & $?$ & $?$ & $?$ & $?$ & \\
\hline
\end{tabular}




\begin{tabular}{|c|c|c|c|c|c|c|c|c|c|c|}
\hline \multirow{2}{*}{\multicolumn{2}{|c|}{ Study }} & \multicolumn{2}{|c|}{ Steroids } & \multirow{2}{*}{$\begin{array}{l}\text { Mac- } \\
\text { rolides/ } \\
\text { Doxycy- } \\
\text { cline }\end{array}$} & \multirow{2}{*}{$\begin{array}{l}\text { Allergic } \\
\text { rhinitis }\end{array}$} & \multirow{2}{*}{$\begin{array}{l}\text { Smok- } \\
\text { ing }\end{array}$} & \multirow{2}{*}{$\begin{array}{l}\text { Anti- } \\
\text { hista- } \\
\text { mines }\end{array}$} & \multirow[t]{2}{*}{ NERD } & \multirow[t]{2}{*}{ Asthma } & \multirow{2}{*}{$\begin{array}{l}\text { Additional } \\
\text { parameters }\end{array}$} \\
\hline & & Local & $\begin{array}{l}\text { Sys- } \\
\text { temic }\end{array}$ & & & & & & & \\
\hline 27 & $\begin{array}{l}\text { Wang et al. } \\
2015^{47}\end{array}$ & + & - & - & $?$ & $?$ & $?$ & $?$ & - & $\begin{array}{l}\text { NP derived nasal epithelial cell } \\
\text { culture }\end{array}$ \\
\hline 28 & $\begin{array}{l}\text { Wang et al. } \\
2015^{48}\end{array}$ & $\#$ & - & - & + & $?$ & $?$ & + & + & $\begin{array}{l}\text { \#Repeated measures after tre- } \\
\text { atment. All pts with eosinophi- } \\
\text { lic CRSwNP }\end{array}$ \\
\hline 29 & Bae et al. $2016^{49}$ & - & - & - & - & $?$ & - & - & - & $\begin{array}{l}\text { No non-steroidal anti-inflam- } \\
\text { matories }\end{array}$ \\
\hline 30 & $\begin{array}{l}\text { Wang et al. } \\
2016^{50}\end{array}$ & $?$ & - & - & + & $?$ & $?$ & $?$ & + & $\begin{array}{l}\text { Significant differences in age } \\
\text { and prior surgery }\end{array}$ \\
\hline 31 & $\begin{array}{l}\text { Homma et al. } \\
2017^{51}\end{array}$ & $?$ & $?$ & $?$ & + & $?$ & $?$ & $?$ & + & \\
\hline 32 & Kim et al. $2017^{52}$ & - & - & - & + & $?$ & - & $?$ & $?$ & Fungal CRS pts excluded \\
\hline 33 & Park et al. $2017^{53}$ & - & - & - & - & $?$ & - & - & + & $\begin{array}{l}\text { NP derived fibroblasts No } \\
\text { antileukotrienes }\end{array}$ \\
\hline 34 & Shin et al. $2017^{54}$ & - & - & - & + & $?$ & - & & & \\
\hline 35 & Yang et al. $2017^{55}$ & - & - & - & - & + & - & & - & Fungal CRS excluded \\
\hline 36 & $\begin{array}{l}\text { Chen et al. } \\
2018^{56}\end{array}$ & - & - & - & + & + & & & + & Fungal CRS excluded \\
\hline 37 & $\begin{array}{l}\text { Sugimoto et al. } \\
2018^{57}\end{array}$ & - & - & - & $?$ & $+^{*}$ & & $+{ }^{*}$ & $+{ }^{*}$ & $\begin{array}{l}\text { Non-eosinophilic NP Fungal } \\
\text { CRS excluded }\end{array}$ \\
\hline 38 & $\begin{array}{l}\text { Wang et al. } \\
2018^{58}\end{array}$ & - & - & - & - & $?$ & & & - & $\begin{array}{l}\text { Dispersed nasal polyp cells No } \\
\text { antileukotrienes }\end{array}$ \\
\hline 39 & $\begin{array}{l}\text { Zhang et al. } \\
2018^{59}\end{array}$ & - & - & $?$ & - & & & - & - & $\begin{array}{l}\text { Exosomes isolated from nasal } \\
\text { lavage fluid }\end{array}$ \\
\hline 40 & Kim et al. $2019^{60}$ & - & - & - & + & $T$ & - & - & - & Fungal CRS excluded \\
\hline 41 & $\begin{array}{l}\text { Xiang et al. } \\
2019^{13}\end{array}$ & - & - & $?$ & & & & - & + & Recurrent cases included \\
\hline 42 & Yan et al. $2019^{61}$ & - & - & $?$ & + & ? & $?$ & - & + & $\begin{array}{l}\text { No pts with fungal CRS \& } \\
\text { infiltrating neutrophils. No } \\
\text { anti-leukotrienes }\end{array}$ \\
\hline 43 & $\begin{array}{l}\text { Vetushi et al. } \\
2020^{62}\end{array}$ & $?$ & ? & & & + & $?$ & $?$ & $?$ & No anti-platelet/ anticoagulant \\
\hline
\end{tabular}

NERD: nonsteroidal anti-inflammatory drug exacerbated respiratory disease, NP: nasal polyp, CRS: chronic rhinosinusitis, NS: non-significant, pts: patients. Notes were marked with * when the authors commented on the differences in the numbers of patients being non-significant between subgroups. In vitro studies (cell cultures) are highlighted with grey.

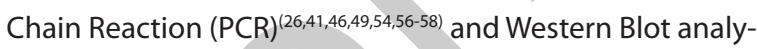
$\operatorname{sis}^{(13,29,46,49,53,54,57,59,62)}$ were also selected by several researchers, while immunofluorescence ${ }^{(27,34,37,57)}$, Northern blot analysis(23) and Luminex ${ }^{(60,61)}$ were used in a minority of studies. It is however of note that in studies where multiple methods were applied (quantitative) m-RNA and protein levels were reported to be in consistency with each other and with semi-quantitative or qualitative immunohistochemical results ${ }^{(26,34,41,49,57,62)}$.

Most of the present literature refers to tissue homogenates, where mechanical micro-disruption of fresh tissue permeabilised the cell membranes in order to extract mRNA and proteins. One third of the researchers have conducted a detailed topographic analysis of their results $(22,23,26,28,31,33,36,38,39,41,43,49,51,52,57,6$ 2). There are also few studies on cell cultures (fibroblasts and/or epithelial cells) originating from patient populations $>10$ (Table
$1)^{(23,47,53,58)}$. Only three studies attempted to assess MMP molecular and activation forms $s^{(22,27,29)}$. By the use of Western blotting it was found that in re-operated patients, the proportion of latent mesenchymal pro-MMP-8 isoform was significantly lower than in patients who had not been previously operated ${ }^{(27)}$. The major MMP-8 form observed in nasal lavages from controls was the latent one ${ }^{(27)}$. By the use of zymographyin NP tissue homogenates, CRSwNP patients were reported to show greater MMP-9 activity than controls, while no differences were found in the enzymatic activity of MMP-2(29).

\section{Risk of bias within studies}

Allergic rhinitis, nonsteroidal anti-inflammatory drug exacerbated respiratory disease (NERD) and asthma, when not reported otherwise, were consistently over-expressed in CRSwNP patients 
Table 2. Heterogeneity of study samples in studies comparing CRSwNP patients with controls and/or CRSsNP patients (39 studies).

\begin{tabular}{|c|c|c|c|}
\hline \multirow{2}{*}{ CRSwNP } & \multicolumn{2}{|l|}{ Samples } & \multirow[t]{2}{*}{ Number of studies } \\
\hline & controls & CRSsNP & \\
\hline NP & IT & - & $\operatorname{Ten}^{22,23,26,38,39,42,43,49,54,58}$ \\
\hline NP & IT & EM & Five $^{34,36,41,47,50}$ \\
\hline NP & IT & OMCM & $\mathrm{Two}^{37,56}$ \\
\hline NP & IT, UP & - & One $e^{61}$ \\
\hline NP & IT, UP & UP & One $e^{13}$ \\
\hline NP & IT, UP, EM & UP, EM & One $e^{55}$ \\
\hline NP & MT & - & Three $e^{30,33,40}$ \\
\hline NP & MT & MT & One $e^{28}$ \\
\hline NP & $\mathrm{T}$ & - & One $e^{29}$ \\
\hline NP & NM & - & One $e^{46}$ \\
\hline NP, UP & UP & UP & Three ${ }^{51,53,60}$ \\
\hline NP, UP & UP & - & One $e^{52}$ \\
\hline NP & UP & - & One $e^{57}$ \\
\hline NP & MM & - & One $e^{31}$ \\
\hline NP & $?$ & - & $\mathrm{Two}^{24,62}$ \\
\hline Mucus & NLF & - & One $e^{27}$ \\
\hline Nasal secretions & - & Nasal secretions & One $e^{25}$ \\
\hline \multicolumn{2}{|c|}{ Nasal secretions, serum } & - & One $e^{45}$ \\
\hline \multicolumn{2}{|l|}{ NLF exosomes } & - & One $e^{59}$ \\
\hline \multicolumn{3}{|c|}{ Plasma in allergic and non-allergic NP } & One $\mathrm{e}^{32}$ \\
\hline
\end{tabular}

CRSwNP: chronic rhinosinusitis with nasal polyposis, CRSsNP: chronic rhinosinusitis without nasal polyposis, NP: nasal polyp, IT: inferior turbinate, MT: middle turbinate, UP: uncinate process, NM: nasal mucosa, EM: ethmoid sinus mucosa, MM: maxillary sinus mucosa, OMCM: osteomeatal complex mucosa, intraop: intra-operatively, NLF: nasal lavage fluid.

compared to controls (Table 1). Most of the studies clearly reported the discontinuation of steroids (local and systemic), antihistamines, macrolides and doxycycline in the patients included. Information on prior nasal surgeries was not provided by most of the manuscripts.

\section{Results of individual studies}

The main primary outcomes are presented in Table 3. TIMP-3 was reported non-detectable at least by the methods applied by Li et al. (2010) ${ }^{(34)}$. The effects of the various treatments on MMPs are summarized in Table 4. Table 5 summarizes the results of studies on eosinophilic and non-eosinophilic subpopulations. Histological localization of MMPs has attributed interesting results. Microscopic examination was indicative of important MMP-1 involvement in ECM degradation ${ }^{(36,39)}$, whereas its presence in the NP epithelium was debated ${ }^{(23,36,39,51)}$. MMP-2 was found mainly in the NP epithelial cells and to a lesser extent in submucosal glands, vessels, inflammatory cells and connective tissue, with studies attempting to spot any differences in its localization between CRSwNP and controls or CRSsNP being inconclusive ${ }^{(26,28,31,36,38,39)}$. MMP-9 was also found in multiple tissue structures ${ }^{(22,26,28,31,38,39)}$, with staining being stronger in the epithelium than the stroma ${ }^{(43)}$. Most researchers agree that NP samples exhibited incr.eased MMP-9 in surface epithelium ${ }^{(22,28,43)}$ vascular endothelial cells ${ }^{(22)}$ infiltrating inflammatory cells ${ }^{(22,28)}$, glands(28), $\mathrm{ECM}^{(28,39)}$ compared to controls. Finally, TIMP-1 was localized in epithelial cells ${ }^{(26,31,39)}$, while its presence in other tissue structures was debated $^{(26,31,39,62)}$. Any differences between CRS patients and controls regarding TIMP-1 are unknown.

\section{Synthesis of results}

MMP-9 and MMP-2 were selected by most researchers as potential biomarkers or target molecules for therapeutical approaches, but comparisons of CRSWNP populations with controls were grossly equally distributed between non-significant and higher results in CRSWNP. MMP-1 and MMP-7 were less frequently selected as target molecules, but consistently found higher in CRSwNP patients than controls. CRSwNP and CRSsNP do not seem to differ significantly as far as MMPs are concerned. Results on TIMP-1 are also inconclusive. None of the studies found TIMP levels to be higher in CRSwNP than controls or CRSsNP. Information on MMP/TIMP ratios is currently limited (Table 3 ). Variation due to heterogeneity across studies on MMP-9 $(22,26,28,29,30,38,40,42,43)$ was estimated as considerable $\left(\chi^{2}=74.27\right.$; $\mathrm{p}<0.00001$ and $\mathrm{I} 2=91 \%)$. For MMP- 2 the respective values were $\chi^{2}=59.72(p<0.00001)$ and $I^{2}=90 \%(22,26,28,29,38,40,43)$, while for TIMP- 1 $\chi^{2}=39.07(p<0.00001)$ and $I^{2}=92 \%(26,28,30,43)$. Further analysis revealed that the laboratory technique used was not the sole factor of heterogeneity, and variation among studies applying the same laboratory tests was still high $\left(1^{2}>90 \%\right)$. Heterogeneity was calculated for the molecules where the largest number of studies were performed, because heterogeneity statistics can be biased when meta-analysis is conducted on a small number of studies ${ }^{(63)}$. Due to these considerable heterogeneity issues further quantitative synthesis of the results was not performed.

\section{Risk of bias across studies}

Besides the differences in the synthesis and characteristics of the study populations (Table 1), samples (Table 2 ) and diagnostic methods, limited numbers of recruited subjects are noted. The numbers of subjects in CRSWNP subpopulations range between 12-191 (median $\pm S D ; 21 \pm 26.4$ ), in controls 6-102 (15 \pm 15.6$)$ and CRSsNP 10-61 (20 \pm 11.5$)$.

\section{Additional analyses}

No correlations have been established between MMPs and the cytokines selected in this review. A positive correlation between 
Table 3. Presentation of the primary outcomes found in clinical studies. Results refer to tissue samples, unless stated otherwise.

\begin{tabular}{|c|c|c|c|c|c|}
\hline \multirow[t]{2}{*}{ Molecules } & \multicolumn{2}{|c|}{ CRSwNP vs controls } & \multicolumn{3}{|c|}{ CRSwNP vs CRSsNP } \\
\hline & Higher & NS & Higher & Lower & NS \\
\hline MMP-1 & $\begin{array}{l}\text { Eyibilen 2011, }{ }^{36} \text { De Borja Callejas } 2013 \\
\text { in ECM and eos, }{ }^{39} \text { Malinsky 2013,40 } \\
\text { Homma } 2017 \text { in NP and UP51 }\end{array}$ & none & none & none & $\begin{array}{l}\text { Eyibilen 2011,36 } \\
\text { Homma } 2017^{51}\end{array}$ \\
\hline MMP-2 & $\begin{array}{l}\text { Can } 2008^{28} \text { Eyibilen } 20111^{36} \text { Wang } \\
2012 \text { in epith of rec, }{ }^{38} \text { De Borja Callejas } \\
2013 \text { in eos, }{ }^{39} \text { Malinsky } 2013^{40}\end{array}$ & $\begin{array}{l}\text { Lechapt-Zalcman } 2001,{ }^{22} \\
\text { Chen } 2007,{ }^{26} \text { Eisenberg } \\
2008,{ }^{29} \text { Li } 2010,{ }^{34} \text { Wang } \\
2012 \text { in epith of pri, in gl of } \\
\text { rec \&pri, }{ }^{38} \text { Yeo } 2013 \text { (rec \& } \\
\text { pri) }{ }^{43} \text { Chen } 2018^{56}\end{array}$ & Can $2008^{28}$ & none & $\begin{array}{l}\text { Van Crombruggen } \\
2012,,^{37} \\
\text { Li 2010,34 } \\
\text { Eyibilen } 2011,{ }^{36} \\
\text { Chen } 2018^{56}\end{array}$ \\
\hline MMP-3 & $\begin{array}{l}\text { Van Crombruggen } 2012,{ }^{37} \text { Vetuschi } \\
2020^{62}\end{array}$ & none & none & none & $\begin{array}{l}\text { Van Crombruggen } \\
2012^{37}\end{array}$ \\
\hline MMP-7 & $\begin{array}{l}\text { Li } 2010,,^{34} \text { Van Crombruggen 2012, } \\
\text { De Borja Callejas } 2013 \text { in eos, }{ }^{39} \\
\text { Yang } 2017,{ }^{, 5} \text { Chen } 2018^{56}\end{array}$ & Can $2008^{28}$ & Yang $2017^{55}$ & Can $2008^{28}$ & $\begin{array}{l}\text { Li } 2010,^{34} \text { Van Crom- } \\
\text { bruggen } 2012,1^{37} \\
\text { Chen } 2018^{56}\end{array}$ \\
\hline MMP-8 & Erbek 2010,3 Eyibilen $2011^{36}$ & $\begin{array}{l}\text { Katainen } 2015(\mathrm{sec} \& \\
\text { serum })^{45}\end{array}$ & & non & Eyibilen $2011^{36}$ \\
\hline MMP-9 & 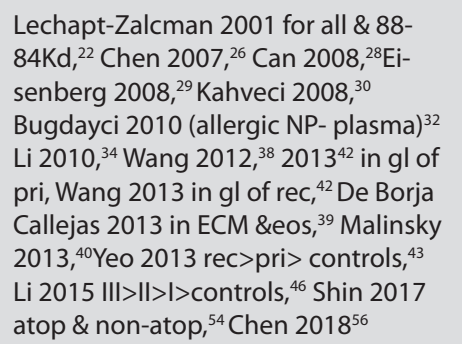 & $\begin{array}{l}\text { Lechapt-Zalcman } 2001 \\
\text { for } 92 \mathrm{kD},{ }^{22} \text { Bugdayci } 2010 \\
\text { (non-allergic NP- plasma), } \\
\text { Van Crombruggen 2012, } \\
\text { Wang 2012, } \\
\text { epith of rec \&pri, Wang } \\
2012 \text { in gl of rec, }{ }^{38} \text { Katainen } \\
2015 \text { (sec \& serum) }{ }^{45}\end{array}$ & & $\begin{array}{l}\text { Watelet } 2004 \\
(\text { sec intraop, } \\
\mathrm{m} 3 \& 6)^{25}\end{array}$ & $\begin{array}{l}\text { Can 2008, } \\
\text { Li 2010, } \\
\text { Van Crombruggen } \\
2012,{ }^{37} \\
\text { Chen } 2018^{56}\end{array}$ \\
\hline Molecules & Higher & NS & Higher & Lower & NS \\
\hline Any ADAM & $\begin{array}{ll}\text { ADAM-33; Erbek } & \text { ADAM-17; Bae } \\
2010,33 \text { ADAM- } & 2016^{49} \\
\text { 10; Zhang 2018 } & \\
\text { (lavage- exoso- }^{\text {mes) }} & \\
\text { m9 } & \end{array}$ & $\begin{array}{l}\text { ADAM-10; Van Crombrug- } \\
\text { gen 2012,37 ADAM-10; Bae } \\
2016^{49}\end{array}$ & none & none & $\begin{array}{l}\text { ADAM-10; } \\
\text { Van Crombruggen } \\
2012^{37}\end{array}$ \\
\hline MMP-9/TIMP-1 & $\begin{array}{ll}\text { Kostamo 2007 } & \text { Katainen } 2015 \\
\text { (pri- mucus), }{ }^{27} & (\mathrm{sec})^{45} \\
\text { Kahveci 2008 } & \end{array}$ & $\begin{array}{l}\text { Kostamo } 2007 \text { (rec- mu- } \\
\text { cus), }{ }^{27} \text { Katainen } 2015 \\
(\text { serum) })^{45}\end{array}$ & none & none & none \\
\hline Molecules & Lower & NS & Higher & Lower & NS \\
\hline TIMP-1 & $\begin{array}{l}\text { Can 2008, }{ }^{28} \text { Kahveci } 2008,{ }^{30} \text { Yeo } 2013 \\
\text { (rec \& pri) }\end{array}$ & $\begin{array}{l}\text { Chen } 2007,{ }^{26} \text { Li } 2010,{ }^{34} \mathrm{De} \\
\text { Borja Callejas } 2013^{39} \text { Katai- } \\
\text { nen } 2015 \text { (sec \& serum), } \\
\text { Vetuschi } 2020 \text { (different } \\
\text { distribution) }{ }^{62}\end{array}$ & none & Li $2010^{34}$ & Can $2008^{28}$ \\
\hline TIMP-2 & Li 2015 (controls>I>II \& IIII) & $\begin{array}{l}\text { Li 2010, }{ }^{34} \text { Eyibilen 2011,36 } \\
\text { Katainen } 2015 \text { (sec \& } \\
\text { serum) }{ }^{45}\end{array}$ & none & Eyibilen $2011^{36}$ & Li $2010^{34}$ \\
\hline MMP-9/TIMP-2 & Katainen $2015(\mathrm{sec})^{45}$ & Katainen 2015 (serum) $^{45}$ & & none & none \\
\hline Molecules & NS & & Higher & Lower & NS \\
\hline TIMP-4 & Li $2010,{ }^{34}$ Yang $2017^{55}$ & & none & Li $2010^{34}$ & Yang $2017^{55}$ \\
\hline MMP-7/TIMP-1 & Kostamo 2007 (pri \& rec- mucus) ${ }^{27}$ & & none & none & none \\
\hline MMP-8/TIMP-1 & Kostamo 2007 (pri \& rec- mucus), ${ }^{27}$ Kata & inen 2015 (sec \& serum) ${ }^{45}$ & none & none & none \\
\hline MMP-8/TIMP-2 & Katainen $2015(\sec \& \text { serum })^{45}$ & & none & none & none \\
\hline
\end{tabular}

NS: non-significant, ECM: extracellular matrix, pri: primary, rec: recurrent, eos: eosinophils: gl: glands, epith: epithelium, sec: nasal secretions, NP: nasal polyp, UP: uncinate process, intraop: intraoperative, m: month, atop: atopic 
Table 4. Effects of the various existing treatment options on metalloproteinases and their inhibitors.

\begin{tabular}{|c|c|c|c|c|c|c|c|c|c|}
\hline Study & pts & Study design & Factor & Time & $\begin{array}{l}\text { Sample } \\
\text { type }\end{array}$ & $\begin{array}{l}\text { Upreg- } \\
\text { ulated } \\
\text { mol- } \\
\text { ecules }\end{array}$ & $\begin{array}{l}\text { Down- } \\
\text { regu- } \\
\text { lated } \\
\text { mol- } \\
\text { ecules }\end{array}$ & $\begin{array}{l}\text { NS } \\
\text { results }\end{array}$ & $\begin{array}{c}\text { MMP } \\
\text { assessment }\end{array}$ \\
\hline \multirow{3}{*}{$\begin{array}{l}\text { Van Zele et al } \\
2010^{35}, \\
\text { Belgium, } \\
\text { Germany, } \\
\text { Netherlands } \\
\text { and Australia }\end{array}$} & 14 & \multirow{3}{*}{$\begin{array}{l}\text { Double blind } \\
\text { placebo-control- } \\
\text { led Comparisons } \\
\text { only between } \\
\text { treatment } \\
\text { groups and } \\
\text { placebo }\end{array}$} & $\begin{array}{l}\text { oral methylprednisolone } \\
\text { ( } 32 \mathrm{mg} / \mathrm{d} \text { on days } 1-5 ; 16 \\
\mathrm{mg} / \mathrm{d} \text { on days } 6-10 ; \text { and } 8 \\
\mathrm{mg} / \mathrm{d} \text { on days } 11-20)\end{array}$ & $12 \mathrm{w}$ & \multirow[t]{3}{*}{$\begin{array}{l}\text { Nasal } \\
\text { secreti- } \\
\text { ons }\end{array}$} & & & MMP-9 & \multirow[t]{3}{*}{$\begin{array}{l}\text { ELISA (R\&D } \\
\text { Quantikine; } \\
\text { Minneapolis, } \\
\text { MN) }\end{array}$} \\
\hline & 14 & & $\begin{array}{l}\text { oral doxycycline }(200 \mathrm{mg} \\
\text { on day } 1,100 \mathrm{mg} / \mathrm{d} \text { on } \\
\text { days } 2-20)\end{array}$ & & & & $\begin{array}{l}\text { MMP-9 } \\
(w 1,2)\end{array}$ & $\begin{array}{l}\text { MMP-9 } \\
(w 8,12)\end{array}$ & \\
\hline & 12 & & placebo (lactose) & & & & & MMP-9 & \\
\hline \multirow[t]{2}{*}{$\begin{array}{l}\text { Yigit et al } \\
2011^{15} \text {, Turkey }\end{array}$} & \multirow[t]{2}{*}{27} & \multirow[t]{2}{*}{ Case series } & $\begin{array}{l}\text { Oral methylprednisolone, } \\
\text { starting with } 1 \mathrm{mg} / \mathrm{kg} / \\
\text { day and decreased } 10 \mathrm{mg} \\
\text { every 3rdday, until FESS. }\end{array}$ & $1 \mathrm{~m}$ & \multirow[t]{2}{*}{$\begin{array}{l}\text { Middle } \\
\text { meatus } \\
\text { mucosa }\end{array}$} & & $\begin{array}{l}\text { MMP-2 } \\
\text { TIMP-1 }\end{array}$ & $\begin{array}{l}\text { MMP-9, } \\
\text { MMPs/ } \\
\text { TIMP-1 }\end{array}$ & \multirow[t]{2}{*}{$\begin{array}{l}\text { ELISA (Ray- } \\
\text { Biotec, Inc., } \\
\text { Norcross, GA; } \\
\text { Human) }\end{array}$} \\
\hline & & & $\begin{array}{l}\text { FESS. No steroids after } \\
\text { FESS }\end{array}$ & $1 \mathrm{~m}$ & & & & $\begin{array}{l}\text { MMP-9, } \\
\text { MMPs/ } \\
\text { TIMP-1 }\end{array}$ & \\
\hline $\begin{array}{l}\text { Wang et al } \\
2015^{48} \text {, China }\end{array}$ & 60 & $\begin{array}{l}\text { Double blind } \\
\text { placebo- } \\
\text { controlled study } \\
\text { on eosinophilic } \\
\text { CRSwNP }\end{array}$ & $\begin{array}{l}1 \mathrm{mg} \text { of budesonide trans- } \\
\text { nasal nebulization twice } \\
\text { daily for } 14 \text { days }\end{array}$ & $\begin{array}{l}14 \text { days } \\
\text { Before vs } \\
\text { after } \\
\text { treatment }\end{array}$ & NP & $\begin{array}{l}\text { TIMP-1 } \\
\text { TIMP-2 } \\
\text { TIMP-4 }\end{array}$ & $\begin{array}{l}\text { MMP-2 } \\
\text { MMP-7 } \\
\text { MMP-8 } \\
\text { MMP-9 }\end{array}$ & TIMP-3 & $\begin{array}{l}\text { Immuno- } \\
\text { assay Flu- } \\
\text { orokine MAP } \\
\text { Multiplex } \\
\text { (R\&D MN) }\end{array}$ \\
\hline \multirow[t]{3}{*}{$\begin{array}{l}\text { de Borja } \\
\text { Callejas et al } \\
2015^{44} \text {, Spain }\end{array}$} & \multirow[t]{3}{*}{18} & \multirow[t]{3}{*}{ Case series } & \multirow[t]{3}{*}{$\begin{array}{l}\text { Oral prednisone ( } 30 \mathrm{mg} \\
\text { daily for } 4 \text { days followed by } \\
\text { a } 2 \text {-day tapered reduction } \\
\text { of } 5 \mathrm{mg} \text { ) } \\
\text { and intranasal budesonide } \\
\text { ( } 400 \mathrm{mg} / \text { twice a day) for } 2 \\
\text { weeks, followed } \\
\text { by intranasal budesonide } \\
\text { alone for } 10 \text { additional } \\
\text { weeks. }\end{array}$} & \multirow[t]{3}{*}{$\begin{array}{l}\text { (w0) after } 2 \\
\text { weeks (w2) } \\
\text { and } 12 \\
\text { weeks (w12) }\end{array}$} & $\begin{array}{l}\text { NP } \\
\text { epithe- } \\
\text { lium } \\
\text { NP } \\
\text { glands } \\
\text { NP } \\
\text { vessels }\end{array}$ & \multirow[t]{3}{*}{$\begin{array}{l}\text { MMP-9; } \\
\text { w0-w2 }\end{array}$} & & $\begin{array}{l}\text { MMP-7 } \\
\text { TIMP-1 } \\
\text { MMP- } \\
1,2,7,9 \\
\text { TIMP-1 } \\
\text { MMP- } \\
1,2,7,9 \\
\text { TIMP-1 }\end{array}$ & \multirow[t]{3}{*}{$\begin{array}{l}\text { Ab MMP-1, } \\
-2,-7,-9, \\
\text { and TIMP-1 } \\
\text { with mouse } \\
\text { monoclonal } \\
\text { anti-human } \\
\text { (Calbiochem; } \\
\text { Merck, } \\
\text { Darmstadt, } \\
\text { Germany). }\end{array}$} \\
\hline & & & & & NP ECM & & $\begin{array}{l}\text { MMP-9; } \\
\text { w2-w12 }\end{array}$ & $\begin{array}{l}\text { MMP- } \\
1,2,7 \\
\text { MMP-9; } \\
\text { w0-w2 } \\
\text { TIMP-1 }\end{array}$ & \\
\hline & & & & & $\begin{array}{l}\text { eosino- } \\
\text { phils }\end{array}$ & & $\begin{array}{l}\text { MMP- } \\
1,7,9 \\
\text { MMP-2; } \\
\text { w2-w12 }\end{array}$ & $\begin{array}{l}\text { MMP-2; } \\
\text { w0-w2 } \\
\text { TIMP-1 }\end{array}$ & \\
\hline
\end{tabular}

NP: nasal polyp, MMP: metalloproteinase, TIMP: tissue inhibitor of metalloproteinase, CRSwNP: chronic rhinosinusitis with nasal polyposis, w: week, NS: non-significant, IL: interleukin, MPO: myeloperoxidase, TGF $\beta$ : Transforming growth factor $\beta$, ECP: Eosinophil Cationic Protein, IFN: interferon, IgE: immunoglobulin E, Ab: antibodies, FESS: functional endoscopic sinus surgery, preop: preoperatively, postop: postoperatively, Immhisto: immunohistochemistry, ELISA: enzyme-linked immunosorbent assay.

TIMP-1 and IL-5 has only been noted in a western European population ${ }^{(26)}$. Neither was the expression of MMP-1, MMP-2, MMP-9, ADAM-33 and ADAM-like decysin-1 correlated to polyp grade or Lund-Mackay radiologic score ${ }^{(33,38,40)}$. In one study, tissue MMP-2/TIMP-1 ratios were positively correlated with postmedication endoscopic, post-medication $\mathrm{CT}$ and post-surgical endoscopic scores. MMP-9/TIMP-1 ratios were positively cor- related only with post-medication CT scores. TIMP-1 concentrations were negatively correlated with post-medication CT and post-surgical endoscopic scores ${ }^{(15)}$. Low preoperative MMP-9 levels in nasal secretions were significantly associated with good postoperative healing and high MMP-9 concentrations in the late postoperative period were related to poor healing in terms of endoscopic scores ${ }^{(25)}$. 
Table 5. Summary of results presented by studies comparing eosinophilic and non-eosinophilic subpopulations.

\begin{tabular}{|c|c|c|c|}
\hline \multirow[t]{2}{*}{ Molecules } & \multicolumn{2}{|c|}{ CRSwNP vs controls; Eosinophilic/non-eosinophilic } & CRSwNP vs CRSsNP; Eosinophilic/non-eosinophilic \\
\hline & Same results & Different results & Different results \\
\hline MMP-1 & {$[+/+]$ Kim $2019^{60}$ Yan $2019^{61}$} & {$\left[+\right.$, NS] $\operatorname{Kim} 2017^{52}$} & {$[+/+] \operatorname{Kim} 2019^{60}$} \\
\hline MMP-2 & $\begin{array}{l}\text { [NS/NS] Kim } 2019^{60} \text { Yan } 2019^{61}[-/-] \\
\text { Shi } 2013^{41}\end{array}$ & [NS/+] Kim $2017^{52}$ & [NS/NS] Kim $2019^{60}$ \\
\hline MMP-3 & {$[+/+]$ Yan $2019^{61}$} & [+/NS] Kim $2019^{60}$ & [NS/NS] Kim $2019^{60}$ \\
\hline MMP-7 & {$[+/+]$ Shi $2013^{41}[N S / N S]$ Yan $2019^{61}$} & & [NS/NS] Shi $2013^{41}$ \\
\hline MMP-8 & & {$[N S /+]$ Yan $2019^{61}$} & \\
\hline MMP-9 & $\begin{array}{l}{[+/+] \text { Shin } 2017^{54} \text { Wang } 2018^{58}} \\
\text { Kim } 2019^{60}\left[\text { NS/NS] Yan } 2019^{61}\right.\end{array}$ & [NS/+] Kim $2017^{52}$ & {$[\mathrm{NS} /+] \mathrm{Kim} 2019^{60}$} \\
\hline DEC1 & {$[+/+]$ Sugimoto $2018^{57}$} & & \\
\hline TIMP-1 & $\begin{array}{l}\text { [NS/NS] Shi } 2013^{41} \text { Shin } 2017^{54}[-/-] \\
\text { Yan } 2019^{61}\end{array}$ & $\begin{array}{l}\text { [NS/-] Kim } 2017^{52} \\
\operatorname{Kim} 2019^{60}\end{array}$ & $\begin{array}{l}\text { [NS/NS] Shi } 2013^{41} \\
\text { Kim 201960 }\end{array}$ \\
\hline TIMP-2 & [NS/NS] Yan $2019^{61}$ & & \\
\hline TIMP-4 & [NS/NS] Yan $2019^{61}$ & {$[-/ N S]$ Shi $2013^{41}$} & $2013^{41}$ \\
\hline MMP-1/TIMP-1 & {$[+/+] \operatorname{Kim} 2019^{60}$} & & {$[+/+] \operatorname{Kim} 2019^{60}$} \\
\hline MMP-2/TIMP-1 & & [NS/+] Kim $2019^{60}$ & [NS/+] Kim $2019^{60}$ \\
\hline MMP-3/TIMP-1 & {$[+/+] \operatorname{Kim} 2019^{60}$} & & 5/NS] Kim $2019^{60}$ \\
\hline MMP-7/TIMP-1 & [NS/NS] Kostamo $2007^{27}$ & & \\
\hline MMP-8/TIMP-1 & & [NS/+] Kostamo $2007^{27}$ & \\
\hline MMP-9/TIMP-1 & {$[+/+] \operatorname{Kim} 2019^{60}$} & {$[\mathrm{NS} /+]$ Kostamo $2007^{27}$} & [NS/+] Kim $2019^{60}$ \\
\hline
\end{tabular}

MMP: metalloproteinase, TIMP: tissue inhibitor of metalloproteinase, CRSWNP: chronic rhinosinusitis with nasal polyposis, CRSsNP: chronic rhinosinusitis without nasal polyposis, NS: non-significant, DEC: metalloproteinase ADAM-like decysin 1.

\section{Discussion}

\section{Summary of evidence}

Published results are summarized in Tables 3 and 4. However, the strength of evidence is compromised by the heterogeneity of patient/control populations regarding several factors which may independently manipulate MMP levels, the relatively small number of patients and controls recruited, the lack of insight into the histological localization or the functional status of the detected MMPs, as well as the heterogeneity of the methods and samples used.

Factors of heterogeneity among study populations which may represent important confounding factors

The concerns which were gradually raised during a long research period refer to independent MMP-affecting factors, as well as any unpredictable interactions between them (Table 1). Evidence for the significant inconsistency among studies and the importance of these factors is given by the observed $I^{2}$ and $\chi^{2}$ values.

\section{Allergic rhinitis (AR)}

The prevalence of IgE-mediated allergy to environmental allergens in patients with CRS (both with and without NP) is estimated at $60 \%$ compared with $30 \%$ to $40 \%$ for the general popula- tion $^{(64)}$. Most studies support that AR patients are not suitable for controls in studies investigating MMP-9 $9^{(13,38,65)}, \mathrm{MMP}^{-2^{(13,38)}}$ and MMP-7 levels ${ }^{(13,38)}$. Only one early study conducted in 2001(66) reported no significant differences between patients with perennial AR and controls regarding the levels of MMP-1, $-2,-3$, -9, TIMP-1 and -2 in the nasal mucosa. Most studies also agree that atopic patients attributed significantly increased MMP$9^{(13,31,54)}$, MMP-9/TIMP-1 ${ }^{(13)}$, and MMP-2 ${ }^{(31)}$ levels in NP tissues and nasal secretions ${ }^{(45)}$. Comparable MMP-2,-7,-9 and TIMP-1 levels between NP tissue samples from atopic and non-atopic patients have also been reported ${ }^{(13,26)}$. AR was assessed in a minority of studies through history and skin prick tests, thus allowing for a minor selection bias related to patients with local AR who may thus remain undiagnosed ${ }^{(67)}$.

\section{Origin of tissue samples}

MMP-3 and MMP-9 (but not TIMP-1) were found to be significantly increased in NP samples compared to uncinate process mucosa for both non-eosinophilic and eosinophilic CRSWNP(60). In non-asthmatic patients with AR, NP and maxillary mucosa specimens differed significantly regarding immunohistochemical staining for MMP-2, -9 (higher for NP) and TIMP-1 (lower for $\mathrm{NP}$ ), while in non-allergic non-asthmatic patients differences 
reached significance only for TIMP-1 (lower for NP) ${ }^{(31)}$. Most researchers have chosen mature NP as their study specimens

(Table 2). Comparisons between different tissue specimens may prove to be quite useful in the understanding of the pathophysiology of CRS and are currently lacking.

\section{Primary and recurrent NP}

Most studies provide limited details on the surgical history of their patients. Kostamo et al. ${ }^{(27)}$ reported that in pre-operated patients, the levels of MMP-8/TIMP-1 and MMP-9/TIMP-1 molar ratios in mucus were significantly lower than patients who were operated for the first time, while the MMP-7/TIMP-1 molar ratio was comparable. Yeo et al. ${ }^{(43)}$ found increased MMP-9 expression in the stroma of recurrent NPs compared with primary NPs and controls.

\section{Smoking}

Cigarette smoking seems to be associated with up-regulation of MMP-9 in eosinophilic CRSwNP patients ${ }^{(60)}$ and patients with CRS and asthma ${ }^{(68)}$. Even passive smoking has been found to induce significant increase of MMP-9 expression in nasal secretions in a paediatric population ${ }^{(14)}$.

\section{Pharmaceutical agents}

Few studies have assessed the in vivo effects of various treatments on MMPs in small populations and different target samples (Table 4). In vitro studies have also supported the suppressive action of roxithromycin on MMP-2 and $-9^{(16)}$, of dexamethasone and clarithromycin on MMP-9 ${ }^{(69)}$, and of doxycycline on MMP- $2^{(17)}$. Downregulation of MMPs following corticosteroid or antibiotic treatment, in vivo, may be interpreted not only as a direct inhibitory pharmacological effect ${ }^{(70,71)}$, but also as an indirect by-product of the suppression of the inflammatory process. Additional potential MMP modulators may include simvastatin (in vitro studies) ${ }^{(72)}$, angiotensin converting enzyme inhibitors and angiotensin receptor blockers (case series) ${ }^{(73)}$.

\section{Acute infection}

Acute infections may increase MMP expression ${ }^{(74-77)}$. Although few researchers insisted on ruling out patients with acute nasal infection ${ }^{(26)}$, with most of the study samples being taken during typical elective FESS, we must presume that such cases were excluded.

Most studies have recruited mixed patient populations with different or unspecified presence of comorbidities such asthma and NERD (Table 1). In several studies asthma was not among the exclusion criteria for the recruitment of a "normal control" group either ${ }^{(37,51,54)}$. However, patients with asthma, CRSwNP, or both have been reported to exhibit lower MMP-9, MMP-9/TIMP1 and MMP-9/TIMP-2 levels in nasal secretions and higher MMP-
9, MMP-9/TIMP-1 and MMP-9/TIMP-2 levels in serum compared to controls ${ }^{(45)}$. To the best of our knowledge, the effect of asthma on MMP levels in nasal tissue samples is currently unknown. Information regarding the geographical area where the study was undertaken was pooled in view of the well known differences in the CRSWNP phenotypes and inflammatory patterns ${ }^{(1,50,54,78-84)}$. However, the results summarized in Table 3 suggest that Asian and European populations did not exhibit major differences regarding MMPs and TIMPs, thus confirming the conclusions of Li et al. ${ }^{(34)}$ who reported that in contrast with inflammatory patterns, remodelling patterns are at least similar between Chinese and Caucasian patients. Recent research has indeed emphasized on the remodelling procedure being a dissociated parallel or even primary process, rather than a result of inflammation. The two procedures share a multitude of overlapping pathways, with eosinophils, mast cells and activated neutrophils being among the main sources of MMP-2 and $-9^{(24,58,85,86)}$. Comparisons between eosinophilic and non-eosinophilic CRSwNP populations and controls or CRSsNP, have concluded to similarities rather than differences in most cases (Table 5). On the other hand, two studies that have directly compared their eosinophilic and non-eosinophilic subgroups did recognize some differences ${ }^{(27,55)}$ and further studies are definitely needed. Of note, there seems to be no consensus on the diagnostic criteria for eosinophilic CRSwNP. Proposed cut-off points include $>5$ or $>10$ eosinophils per high-power field ${ }^{(87,88)}$. Kostamo et al. $(2007)^{(27)}$ based the diagnosis of NP eosinophilia on either the percentage of eosinophils being $>80 \%$ of all leukocytes or the presence of clusters of eosinophils in the polyp tissue. Other researchers defined their cases as eosinophilic when the number of eosinophils exceeded $10 \%$ of total infiltrating inflammatory cells in the polyp tissue ${ }^{(48,55,89)}$. A clinical score (JESREC score) system has also been proposed ${ }^{(90)}$.

\section{Common methodological choices}

All existing studies refer to specimens from patients undergoing functional endoscopic sinus surgery (FESS). With the most common indication for surgery being failure of medical treatment to alleviate the symptoms, it is obvious that all these studies refer to nasal polyps mature, chronic and resistant to treatment. Early and mature polyps present, however, substantial histopathological differences ${ }^{(7,91)}$. In order to take a more spherical glimpse into the role of MMPs in the pathophysiology of nasal polypogenesis, prospective studies designed to include NP samples or nasal mucosa from patients with early CRSwNP or patients responding well to medical treatment may be very useful.

\section{Clinical implications}

Most of the existing studies compare patients with NP with controls and CRSsNP patients (Table 3). One study has assessed the role of MMPs within different CRSwNP endotypes ${ }^{(46)}$. Few 
studies fulfilling the inclusion criteria have inquired on the possible use of MMPs as biomarkers of disease severity or prognosis, reaching inconclusive results ${ }^{(15,25,33,38,40)}$. The utility of MMPs in every day clinical practice remains unclear and merits further investigation.

\section{Limitations}

The available studies provide only an estimate of in vivo MMP activity. TIMPs and MMP/TIMP ratios have been measured only in a minority of studies (Table 3). Furthermore, only three studies have investigated the forms, active or latent, in which MMPs are found ${ }^{(22,27,29)}$. MMPs are secreted as inactive zymogens and are activated by several different mechanisms ${ }^{(6)}$. MMP measuring and localization by immunohistochemistry, western blot, or ELISA include zymogen and active enzyme ${ }^{(92)}$, while commercially available MMP antibodies may differ considerably regarding their immunohistochemical sensitivity and specificity. Gel zymography which was used in 3 studies ${ }^{(22,27,29)}$ can distinguish between active and zymogen forms based on their migration through the gel ${ }^{(93,94)}$. However, this technique is only suitable for obtaining qualitative or semi-quantitative data, while reliable quantitative data collection through this method is challenging. Furthermore, preceding electrophoresis is known to dissociate TIMPs from MMPs while inducing unpredictable activation of pro-MMPs ${ }^{(75,95)}$. Consequently, the method provides an estimate of the maximum MMP potential rather than the actual in vivo activity ${ }^{(95)}$. Substrate assays, on the other hand, provide quantitative data, but preceding tissue homogenization may compromise in vivo activity estimates by artificially mixing enzymes and inhibitors ${ }^{(96,97)}$. Assays able to detect the native enzyme in solution or after its immobilization by the specific antibody, followed by incubation with labelled substrate may provide a better MMP-activity estimate ${ }^{(75,98)}$.

\section{Conclusion}

The present review has revealed that there are several population and methodology related confounding factors, which may modulate measured MMP levels, and may, at least to some extent, be responsible for the apparently contradictory and inconclusive results found in the literature, regarding MMP expression in CRS. The current literature seems to advocate for remodeling pattern similarities among CRSwNP endotypes with different inflammation patterns. Additional studies are needed in order to assess the roles of MMPs in CRSwNP pathophysiology, comprehend the sources of the apparently contradictory results and investigate their possible utility as biormarkers and/ or therapeutical targets.

\section{Authorship contribution}

SL: Literature collection and review of the literature, data analysis, review of the manuscript. GD: Literature collection, data analysis, review of the manuscript. KG: Literature collection, data analysis, review of the manuscript. MR: Review of the literature, data analysis, preparation and final review of the manuscript.

\section{Conflict of interest}

No conflict of interest.

\section{Financial disclosure}

No funding.

\section{References}

1. Tomassen P, Vandeplas G, Van Zele T, et al. Inflammatory endotypes of chronic rhinosinusitis based on cluster analysis of biomarkers. J Allergy Clin Immunol. 2016; 137(5): 1449-1456.e4

2. Liao B, Liu JX, Li ZY, et al. Multidimensiona endotypes of chronic rhinosinusitis and their association with treatment outcomes. Allergy. 2018; 73(7): 1459-1469.

3. Arpino V, Brock M, Gill SE. The role of TIMPs in regulation of extracellular matrix proteolysis. Matrix Biol. 2015; 44-46: 247-254.

4. Klein T, Bischoff R. Physiology and pathophysiology of matrix metalloproteases. Amino Acids. 2011; 41(2): 271-290

5. Hadler-Olsen E, Fadnes B, Sylte I, UhlinHansen L, Winberg JO. Regulation of matrix metalloproteinase activity in health and disease. FEBS J. 2011; 278(1): 28-45.

6. Löffek S, Schilling O, Franzke CW. Series "matrix metalloproteinases in lung health and disease": Biological role of matrix metalloproteinases: a critical balance. Eur Respir J. 2011; 38(1): 191-208.

7. Schleimer RP. Immunopathogenesis of
Chronic Rhinosinusitis and Nasal Polyposis. Annu Rev Pathol. 2017; 12: 331-357.

8. Bourboulia D, Stetler-Stevenson WG. Matrix metalloproteinases (MMPs) and tissue inhibitors of metalloproteinases (TIMPs): positive and negative regulators in tumor cell adhesion. Semin Cancer Biol. 2010; 20: 161-168.

9. Moher D, Shamseer L, Clarke M, et al. Preferred reporting items for systematic review and meta-analysis protocols (PRISMA-P) 2015 statement. Syst Rev. 2015; 4(1): 1.

10. Won HK, Kim YC, Kang MG, et al. Agerelated prevalence of chronic rhinosinusitis and nasal polyps and their relationships with asthma onset. Ann Allergy Asthma Immunol 2018; 120: 389-94.

11. Daniel WW. Biostatistics: A Foundation for Analysis in the Health Sciences. Determination of sample size for estimating means. 7th edition. New York: John Wiley \& Sons, 1999; 198-190.

12. Walsh GM. The anti-inflammatory effects of levocetirizine--are they clinically relevant or just an interesting additional effect?.Allergy
Asthma Clin Immunol. 2009; 5(1): 14

13. Xiang R, Zhang QP, Zhang W, et al. Different effects of allergic rhinitis on nasal mucosa remodeling in chronic rhinosinusitis with and without nasal polyps. Eur Arch Otorhinolaryngol. 2019; 276(1): 115-130.

14. De S, Leong SC, Fenton JE, Carter SD, Clarke RW, Jones AS. The effect of passive smoking on the levels of matrix metalloproteinase 9 in nasal secretions of children. Am J Rhinol Allergy 2011; 25: 226-230.

15. Yigit $O$, Acioğlu E, Gelişgen R, Server EA, Azizli $E$, Uzun $H$. The effect of corticosteroid on metalloproteinase levels of nasal polyposis. Laryngoscope. 2011; 121(3): 667-673.

16. Kanai K, Asano K, Hisamitsu T, Suzaki H. Suppression of matrix metalloproteinase production from nasal fibroblasts by macrolide antibiotics in vitro. Eur Respir J. 2004 23(5): 671-678

17. Shin JM, Park JH, Kang B, Lee SA, Park IH, Lee HM. Effect of doxycycline on transforming growth factor-beta-1-induced matrix metalloproteinase 2 expression, migration, and collagen contraction in nasal polyp-derived fibroblasts. Am J Rhinol Allergy. 2016; 30(6): 
385-390.

18. Nohr EA, Liew Z. How to investigate and adjust for selection bias in cohort studies. Acta Obstet Gynecol Scand. 2018; 97(4): 407-416.

19. Gagliani N, Huber S. Basic Aspects of T Helper Cell Differentiation. Methods Mol Biol. 2017; 1514: 19-30.

20. Baba S, Kagoya R, Kondo K, Suzukawa M, Ohta K, Yamasoba T. T-cell phenotypes in chronic rhinosinusitis with nasal polyps in Japanese patients. Allergy Asthma Clin Immunol. 2015; 11: 33.

21. Bedoya SK, Lam B, Lau K, Larkin J 3rd. Th17 cells in immunity and autoimmunity. Clin Dev Immunol. 2013; 2013: 986789.

22. Lechapt-Zalcman E, Coste A, d'Ortho MP Frisdal E, Harf A, Lafuma C, Escudier E. Increased expression of matrix metalloproteinase-9 in nasal polyps. J Pathol. 2001; 193(2): 233-241.

23. Liu CM, Hong CY, Shun CT, et al. Matrix metalloproteinase-1 and tissue inhibitor of metalloproteinase-1 gene expressions and their differential regulation by proinflammatory cytokines and prostaglandin in nasal polyp fibroblasts. Ann OtolRhinolLaryngol. 2001; 110(12): 1129-1136

24. Lee YM, Kim SS, Kim HA, et al. Eosinophil inflammation of nasal polyp tissue: relationships with matrix metalloproteinases, tissue inhibitor of metalloproteinase-1, and transforming growth factor-beta1. J Korean Med Sci. 2003; 18(1): 97-102.

25. Watelet JB, Annicq B, van Cauwenberge P, Bachert C. Objective outcome after functional endoscopic sinus surgery: prediction factors. Laryngoscope. 2004; 114(6): 10921097

26. Chen YS, Langhammer $T$, Westhofen $M$, Lorenzen J. Relationship between matrix metalloproteinases MMP-2, MMP-9, tissue inhibitor of matrix metalloproteinases-1 and IL-5, IL-8 in nasal polyps. Allergy. 2007; 62(1): 66-72.

27. Kostamo K, Tervahartiala T, Sorsa T, Richardson M, Toskala E. Metalloproteinase function in chronic rhinosinusitis with nasal polyposis. Laryngoscope. 2007; 117(4): 638643.

28. Can IH, Ceylan K, Caydere M, Samim EE, Ustun $H$, Karasoy DS. The expression of MMP-2, MMP-7, MMP-9, and TIMP-1 in chronic rhinosinusitis and nasal polyposis. Otolaryngol Head Neck Surg. 2008; 139(2): 211-215.

29. Eisenberg G, Pradillo J, Plaza G, Lizasoain I, Moro MA. Increased expression and activity of MMP-9 in chronic rhinosinusitis with nasal polyposis. Acta Otorinolaringol Esp 2008; 59: 444-447.

30. Kahveci OK, Derekoy FS, Yilmaz M, Serteser M, Altuntas A. The role of MMP-9 and TIMP1 in nasal polyp formation. Swiss Med Wkly. 2008; 138(45-46): 684-688.

31. Shin HW, Han DH, Lim YS, et al. Nonasthmatic nasal polyposis patients with allergy exhibit greater epithelial MMP posi- tivity. Otolaryngol Head Neck Surg. 2009; 141(4): 442-447

32. Bugdayci G, Kaymakci M, Bukan N. Matrix metalloproteinase-9 (MMP-9) in allergic nasal polyps. Acta Histochem. 2010; 112(1): 92-95.

33. Erbek SS, Erinanc H, Erbek S, Topal O, Kiyici $\mathrm{H}$. Expression of a disintegrin and metalloproteinase 33 protein in nasal polyposis: An immunohistochemical study. Am J Rhinol Allergy 2010; 24: 7982

34. Li X, Meng J, Qiao X, et al. Expression of TGF, matrix metalloproteinases, and tissue inhibitors in Chinese chronic rhinosinusitis. J Allergy Clin Immunol. 2010; 125(5): 1061 1068

35. Van Zele T, Gevaert P, Holtappels G, et al Oral steroids and doxycycline: two different approaches to treat nasal polyps. J Allergy Clin Immunol. 2010; 125(5): 1069-1076.

36. Eyibilen A, Cayli S, Aladag I, Koç S, Gurbuzler L, Atay GA. Distribution of matrix metalloproteinases MMP-1, MMP-2, MMP-8 and tissue inhibitor of matrix metalloproteinases-2 in nasal polyposis and chronic rhinosinusitis. Histol Histopathol. 2011; 26(5): 615-621.

37. Van Crombruggen K, Holtappels G, De Ruyck N, Derycke L, Tomassen P, Bachert C. RAGE processing in chronic airway conditions: involvement of Staphylococcus aureus and ECP. J Allergy Clin Immunol. 2012; 129(6): 1515-1521.

38. Wang LF, Chien CY, Chiang FY, Chai CY, Ta CF. Corelationship between matrix metalloproteinase 2 and 9 expression and severity of chronic rhinosinusitis with nasal polyposis. Am J Rhinol Allergy. 2012; 26(1): e1-4.

39. de Borja Callejas F, Picado C, MartínezAntón A, et al. Differential expression of remodeling markers by tissue structure in nasal polyposis. Am J Rhinol Allergy. 2013; 27(3): e69-e74.

40. Malinsky RR, Valera FC, Cavallari FE, et al Matrix metalloproteinases and their impact on sinusal extension in chronic rhinosinusitis with nasal polyps. Eur Arch Otorhinolaryngol. 2013; 270(4): 1345-1348.

41. Shi $L L$, Xiong $P$, Zhang $L$, et al. Features of airway remodeling in different types of Chinese chronic rhinosinusitis are associated with inflammation patterns. Allergy. 2013; 68(1): 101-109.

42. Wang LF, Chien CY, Chiang FY, Chai CY, Ta CF. Expression of matrix metalloproteinase-2 and matrix metalloproteinase- 9 in recurrent chronic rhinosinusitis with nasal polyposis. Kaohsiung J Med Sci. 2013; 29(1): 26-31.

43. Yeo NK, Eom DW, Oh MY, Lim HW, Song YJ. Expression of matrix metalloproteinase 2 and 9 and tissue inhibitor of metalloproteinase 1 in nonrecurrent vs recurrent nasal polyps. Ann Allergy Asthma Immunol. 2013; 111(3): 205-210

44. de Borja Callejas F, Martínez-Antón A Picado C, et al. Corticosteroid treatment regulates mucosal remodeling in chronic rhinosinusitis with nasal polyps.
Laryngoscope. 2015; 125(5): E158-E167.

45. Katainen E, Kostamo K, Virkkula P, et al. Local and systemic proteolytic responses in chronic rhinosinusitis with nasal polyposis and asthma. Int Forum Allergy Rhinol 2015; 4: 294-302.

46. Li X, Tao Y, Li X. Expression of MMP-9/TIMP-2 in nasal polyps and its functional implications. Int J Clin Exp Pathol 2015; 8: 14556 14561.

47. Wang X, Zhao C, Ji W, Xu Y, Guo H. Relationship of TLR2, TLR4 and tissue remodeling in chronic rhinosinusitis. Int J Clin Exp Pathol. 2015; 8(2): 1199-1212.

48. Wang $C$, Lou $H$, Wang $X$, et al. Effect of budesonide transnasal nebulization in patients with eosinophilic chronic rhinosinusitis with nasal polyps. J Allergy Clin Immunol. 2015; 135(4): 922-929.

49. Bae WY, Park SK, Kim do H, Koh TK, Hur DY, Chueh HW. Expression of ADAM17 and ADAM10 in nasal polyps. Int Forum Allergy Rhinol. 2016; 6(7): 731-736.

50. Wang $X$, Zhang $N$, Bo $M$, et al. Diversity of $\mathrm{TH}$ cytokine profiles in patients with chronic rhinosinusitis: A multicenter study in Europe, Asia, and Oceania. J Allergy Clin Immunol. 2016; 138(5): 1344-1353.

51. Homma T, Kato A, Sakashita M, et al. Potential Involvement of the Epidermal Growth Factor Receptor Ligand Epiregulin and Matrix Metalloproteinase-1 in Pathogenesis of Chronic Rhinosinusitis. Am J Respir Cell Mol Biol. 2017; 57(3): 334-345.

52. Kim DK, Jin HR, Eun KM, et al. The role of interleukin-33 in chronic rhinosinusitis. Thorax. 2017; 72(7): 635-645

53. Park SK, Jin YD, Park YK, et al. IL-25-induced activation of nasal fibroblast and its association with the remodeling of chronic rhinosinusitis with nasal polyposis. PLoS One 2017; 12(8): e0181806.

54. Shin SH, Kim YH, Ye MK, Choi SY. Immunopathologic characteristics of nasal polyps in adult Koreans: A single-center study. Am J Rhinol Allergy. 2017; 31(3): 168173

55. Yang LY, Li X, Li WT, Huang JC, Wang ZY, Huang ZZ, Chang LH, Zhang GH. VY1y $\delta T$ Cells Are Correlated With Increasing Expression of Eosinophil Cationic Protein and Metalloproteinase-7 in Chronic Rhinosinusitis With Nasal Polyps Inducing the Formation of Edema. Allergy Asthma Immunol Res. 2017; 9(2): 142-151.

56. Chen X, Chang L, Li X, et al. TC17/IL-17A Up-Regulated the Expression of MMP-9 via NF-KB Pathway in Nasal Epithelial Cells of Patients With Chronic Rhinosinusitis. Front Immunol. 2018; 9: 2121.

57. Sugimoto $N$, Nakayama $T$, Kasai $Y$, et al. The role of ADAM-like decysin 1 in non-eosinophilic chronic rhinosinusitis with nasal polyps. Acta Otolaryngol. 2018; 138(9): 830836.

58. Wang $H$, Li ZY, Jiang WX, et al. The activation and function of IL-36y in neutrophilic inflammation in chronic rhinosinusitis. $J$ 
Allergy Clin Immunol. 2018; 141(5): 16461658.

59. Zhang W, Zhang J, Cheng L, et al. A disintegrin and metalloprotease 10-containing exosomes derived from nasal polyps promote angiogenesis and vascular permeability. Mol Med Rep. 2018; 17(4): 5921-5927.

60. Kim DK, Eun KM, Kim MK, et al. Comparison Between Signature Cytokines of Nasal Tissues in Subtypes of Chronic Rhinosinusitis. Allergy Asthma Immunol Res. 2019; 11(2): 201-211.

61. Yan B, Wang $Y$, Li Y, Wang C, Zhang L. Inhibition of arachidonate 15-lipoxygenase reduces the epithelial-mesenchymal transition in eosinophilic chronic rhinosinusitis with nasal polyps. Int Forum Allergy Rhinol. 2019; 9(3): 270-280.

62. Vetuschi A, Pompili S, Di Marco GP, et al. Can the AGE/RAGE/ERK signalling pathway and the epithelial-to-mesenchymal transition interact in the pathogenesis of chronic rhinosinusitis with nasal polyps? Eur J Histochem. 2020; 64(1): 3079.

63. von Hippel PT. The heterogeneity statistic I(2) can be biased in small meta-analyses. BMC Med Res Methodol. 2015 Apr 14;15:35. doi: 10.1186/s12874-015-0024-z. PMID: 25880989; PMCID: PMC4410499.

64. Hamilos DL. Chronic rhinosinusitis: epidemiology and medical management. J Allergy Clin Immunol. 2011; 128(4): 693-707.

65. van Toorenenbergen AW, Gerth van Wijk R, Vermeulen AM. Allergen-induced matrix metalloproteinase-9 in nasal lavage fluid. Allergy 1999; 54: 293-294.

66. Shaida A, Kenyon G, Devalia J, Davies RJ, MacDonald TT, Pender SL. Matrix metalloproteinases and their inhibitors in the nasal mucosa of patients with perennial allergic rhinitis. J Allergy Clin Immunol. 2001; 108(5): 791-796.

67. Eguiluz-Gracia I, Pérez-Sánchez N, Bogas G, Campo P, Rondón C. How to Diagnose and Treat Local Allergic Rhinitis: A Challenge for Clinicians. J Clin Med. 2019; 8(7): 1062

68. Huang CC, Wang CH, Wu PW, et al Increased nasal matrix metalloproteinase-1 and -9 expression in smokers with chronic rhinosinusitis and asthma. Sci Rep. 2019; 9(1): 15357.

69. Zeng M, Li ZY, Ma J, et al. Clarithromycin and dexamethasone show similar antiinflammatory effects on distinct phenotypic chronic rhinosinusitis: an explant mode study. BMC Immunol. 2015; 16: 37

70. Nagase H, Woessner JF Jr. Matrix metalloproteinases. J Biol Chem 1999; 274: 21491 21494.

71. Vincenti MP. The matrix metalloproteinase (MMP) and tissue inhibitor of metalloproteinase (TIMP) genes. Transcriptional and post- transcriptional regulation, signal transduction and cell-type specific expression. Methods Mol Biol 2001; 151: 121-148.

72. Park IH, Park SJ, Cho JS, et al. Effect of simvastatin on transforming growth factor beta1-induced myofibroblast differentiation and collagen production in nasal polyp-derived fibroblasts. Am J Rhinol Allergy. 2012; 26(1): 7-11.

73. Brook CD, Maxfield AZ, Stankovic K, Metson RB. The Impact of AngiotensinModulating Antihypertensives on Time Interval to Revision Surgery for Nasal Polyps. Otolaryngol Head Neck Surg. 2016; 155(6): 1046-1052.

74. Atkinson JJ, Senior RM. Matrix metalloproteinase-9 in lung remodeling. Am J Respir Cell Mol Biol. 2003; 28(1): 12-24.

75. Grzela K, Litwiniuk M, Zagorska W, Grzela T. Airway Remodeling in Chronic Obstructive Pulmonary Disease and Asthma: the Role of Matrix Metalloproteinase-9. Arch Immunol Ther Exp (Warsz). 2016; 64(1): 47-55.

76. Wang J, Watanabe S, Matsukura S, Suzaki H. Double-stranded RNA poly(l:C) enhances matrix metalloproteinase mRNA expression in human nasal polyp epithelial cells. Acta Otolaryngol Suppl. 2009; (562): 105-109.

77. Wang JH, Kwon HJ, Jang YJ. Rhinovirus upregulates matrixmetalloproteinase- 2 . matrix metalloproteinase-9, and vascular endothelial growth factor expression in nasal polyp fibroblasts. Laryngoscope. 2009; 119(9): 1834-1838.

78. Bachert C, Akdis CA. Phenotypes and emerging endotypes of chronic rhinosinusitis. J Allergy Clin Immunol Pract. 2016; 4(4): 621-628.

79. Zhang N, Van Zele T, Perez-Novo C, et al. Different types of T-effector cells orchestrate mucosal inflammation in chronic sinus disease. J Allergy Clin Immunol. 2008; 122(5): 961-968

80. Wu D, Wang J, Zhang M. Altered Th17/Treg ratio in nasal polyps with distinct cytokine profile: association with patterns of inflammation and mucosal remodeling. Medicine (Baltimore), 2016; 95(10): e2998.

81. Mahdavinia M, Suh LA, Carter RG, et al Increased noneosinophilic nasal polyps in chronic rhinosinusitis in US second-generation Asians suggest genetic regulation of eosinophilia. J Allergy Clin Immunol. 2015; 135(2): 576-579.

82. Ikeda K, Shiozawa A, Ono N, et al. Subclassification of chronic rhinosinusitis with nasal polyp based on eosinophil and neutrophil. Laryngoscope. 2013; 123(11): E1-E9.

83. Ishitoya J, Sakuma $Y$, Tsukuda M Eosinophilic chronic rhinosinusitis in Japan. Allergol Int. 2010: 59(3): 239-245.

84. Tsuda $T$, Maeda $Y$, Nishide $M$, et al. Eosinophil-derived neurotoxin enhances airway remodeling in eosinophilic chronic rhinosinusitis and correlates with disease severity. Int Immunol. 2019; 31 (1): 33-40.

85. Pawankar R, Nonaka M. Inflammatory mechanisms and remodeling in chronic rhinosinusitis and nasal polyps. Curr Allergy Asthma Rep. 2007; 7(3): 202-208.

86. Kuhar HN, Tajudeen BA, Mahdavinia M, Gattuso P, Ghai R, Batra PS. Inflammatory infiltrate and mucosal remodeling in chron-

ic rhinosinusitis with and without polyps: structured histopathologic analysis. Int Forum Allergy Rhinol. 2017; 7(7): 679-689.

87. Kountakis SE, Arango P, Bradley D, Wade ZK, Borish L. Molecular and cellular staging for the severity of chronic rhinosinusitis. Laryngoscope 2004; 114: 1895-1905.

88. Soler ZM, Sauer D, Mace J, Smith TL. Impact of mucosal eosinophilia and nasal polyposis on quality-of-life outcomes after sinus surgery. Otolaryngol Head Neck Surg 2010; 142: 64-71.

89. Cao PP, Li HB, Wang BF, et al. Distinct immunopathologic characteristics of various types of chronic rhinosinusitis in adult Chinese. J Allergy Clin Immunol 2009; 124: 478-484.

90. Tokunaga T, Sakashita M, Haruna T et al. Novel scoring system and algorithm for classifying chronic rhinosinusitis: the JESREC Study. Allergy 2015; 70: 995-1003.

91. Meng J, Zhou P, Liu Y, et al. The development of nasal polyp disease involves early nasal mucosal inflammation and remodelling. PLOS ONE. 2013; 8(12): e82373.

92. Hadler-Olsen E, Kanapathippillai P, Berg E, Svineng $G$, Winberg JO, Uhlin-Hansen L. Gelatin in situ zymography on fixed, paraffin-embedded tissue: zinc and thanol fixation preserve enzyme activity. J Histochem Cytochem 2010; 58: 29-39.

93. Leber TM, Balkwill FR. Zymography: a single-step staining method for quantitation of proteolytic activity on substrate gels. Anal Biochem 1997; 249: 24-28.

94. Rossano R, Larocca M, Riviello L, et al. Heterogeneity of serum gelatinases MMP-2 and MMP-9 isoforms and charge variants. Cell Mol Med 2014; 18: 242-252.

95. Toth $M$ and Fridman R. Assessment of gelatinases (MMP-2 and MMP-9) by gelatin zymography. Methods Mol Med 2001; 57: 10.

96. Grierson C, Miller D, LaPan P, Brady J. Utility of combining MMP-9 enzyme-linked immunosorbent assay and MMP-9 activity assay data to monitor plasma enzyme specific activity. Anal Biochem 2010; 404: 232-234.

97. Vandooren J, Geurts N, Mertens E, Van den Steen PE, Opdenakker G. Zymography methods for visualizing hydrolytic enzymes. Nat Methods 2013; 10: 211-220.

98. Lauer-Fields J, Sritharan T, Stack, MS, Nagase $H$, Fields GB. Selective hydrolysis of triplehelical substrates by matrix metalloproteinase-2 and -9. J Biol Chem 2003; 278: 18140-18145

Maria Riga, MD, PhD,

ENT Consultant

Department of Otorhinolaryngology

Head and Neck Surgery

Dammam Medical Complex

Dammam, Eastern Province

Kingdom of Saudi Arabia 